\title{
Temporal tuning and attentional gating: Two distinct attentional mechanisms on the perception of rapid serial visual events
}

\author{
Michael S. Ambinder ANd Alejandro Lleras \\ University of Illinois at Urbana-Champaign, Urbana, Illinois
}

\begin{abstract}
We examined the relationship between two different attention limitations on the perception of rapid events: the attentional awakening (AA, an inability to successfully process a target when it appears early on in a rapid stream of events) and the attentional blink ( $\mathrm{AB}$, an inability to successfully process a second target when it appears shortly after a first target [T1]). In four experiments, we failed to find a relationship between the magnitudes of these phenomena. Furthermore, we found two manipulations that selectively modulated the magnitude of each effect without altering the magnitude of the second effect: Expected range of possible rapid serial visual presentation lengths modulated the AA (but not the $\mathrm{AB}$ ), suggesting that the AA reflects an attentional setup cost for perceiving a protracted series of rapid events, whereas the number of possible T1 positions in the stream modulated the magnitude of the $\mathrm{AB}$ (but not the magnitude of the AA). Our results suggest that, despite the surface similarities between the two phenomena, different mechanisms are responsible for these two attentional limitations: Whereas the AA reflects a starting cost associated with the time required to temporally tune attention to the stimulus stream, the $\mathrm{AB}$ reflects a blocking of undesired stimuli, aimed at protecting consolidation of T1 processing.
\end{abstract}

Much research has examined the scope of our ability to select stimuli from our environment for higher level processing. One common approach to uncovering the mechanisms responsible for our ability to selectively attend to various elements of our environment is to examine instances in which our attentional mechanisms fail or perform suboptimally. By determining why and when we go astray, we are able to shed light on how our attentional system manages to extract relevant information from the detailed and complex environment we inhabit. One such investigation of attentional failures looks into how well one can identify two targets (T1 and $\mathrm{T} 2$ ) presented in a stream of distractors, close in time, and at the same location in space. During these rapid serial visual presentation (RSVP) tasks, it is commonly found that identification of T2 is impaired if it is presented near in time to the first target (Raymond, Shapiro, \& Arnell, 1992). This impairment is strongest at around $200 \mathrm{msec}$ after the presentation of $\mathrm{T} 1$ and gradually recovers until baseline identification performance is reached around $500 \mathrm{msec}$ after T1 (a schematic of this finding can be seen in Figure 1). This decrease and recovery of identification accuracy is known as the attentional blink (AB).

There exist two classes of theories regarding the mechanisms responsible for the $\mathrm{AB}$. The deficit in processing $\mathrm{T} 2$ soon after T1 is thought to result from either (1) a reduction in available processing resources for identi- fying T2 (bottleneck models) or (2) a perceptual filter that blocks processing of T2 altogether (filter models). A classic theory of the AB proposed by Chun and Potter (1995) is a two-stage model that argues for limitations in central encoding. Whereas we can perceptually process a vast amount of visual information at any given time, the ensuing representations are short-lived and brittle. In order to protect such representations from decay and from masking by subsequent stimuli, we must protect them by encoding them into short-term memory. The argument is that this transfer from perception to memory (i.e., central encoding) cannot deal with more than one target stimulus at a time (see also Giesbrecht \& Di Lollo, 1998; Jolicœur \& Dell'Acqua, 1998). Within this framework, the AB pattern occurs because of a bottleneck in central encoding; if central encoding is busy processing T1, T2's perceptual representation cannot be encoded into memory before it is masked by subsequent stimuli. Recent work by Dux and colleagues (Dux, Asplund, \& Marois, 2008, 2009; Dux, Ivanoff, Asplund, \& Marois, 2006) has continued to provide evidence in favor of resource depletion accounts of the $\mathrm{AB}$.

A new class of theories about $\mathrm{AB}$ has called into question the claim that a processing limitation is required to account for the phenomenon. These newer theories propose that the $\mathrm{AB}$ is due to the action of perceptual filters, blocking visual stimuli from further processing. In the tempo- 

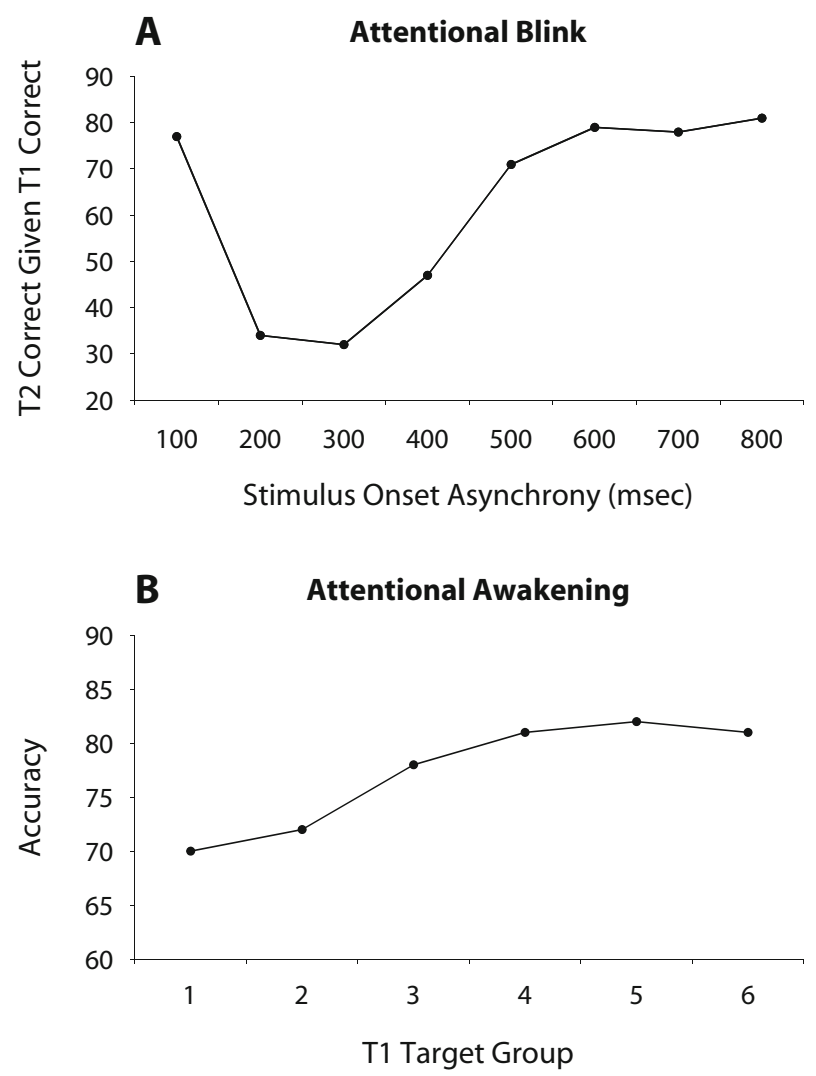

Figure 1. (A) Schematic of the typical attentional blink effect: Accuracy in identifying $T 2$ decreases around $200 \mathrm{msec}$ after the onset of $\mathrm{T} 1$ and recovers to baseline performance around $500 \mathrm{msec}$ after T1 onset. (B) Schematic of the typical attentional awakening effect: Accuracy of single-target identification increases over time and reaches peak performance around 900 msec.

rary loss of control theory, Di Lollo, Kawahara, Ghorashi, and Enns (2005) proposed that the AB might arise because of a temporary loss of control over the perceptual filters that control the access of stimuli to our awareness. In their model, target detection is under top-down control and makes use of a central processor. When a target (T1) is detected, the central processor becomes involved in consolidating the representation and preparing the response to $\mathrm{T} 1$. As a result, it temporarily loses control over the perceptual filters that have been put in place to reject (distractors) or select (targets) stimuli. This loss of control leaves the attentional system vulnerable to distraction. Thus, if a distractor item follows the target, it can exogenously cue the attentional system to attend to a different category of stimuli (distractors) or lead to suboptimal detection of the appropriate target category. This exogenous reorienting of attention to a different category of stimuli is what produces the performance drop-off in detection of 2 . After the central processor has finished preparing the response for T1, it is able to resume endogenous control of the attentional system, and T2 can once again be accurately picked out of the RSVP stream. Olivers and colleagues (Olivers, 2007; Olivers \& Meeter, 2008) recently proposed a second filter model that is in many ways similar to Raymond et al.'s
(1992) original account of the AB. Their model (the boost and bounce model; Olivers \& Meeter, 2008) is based on two simple premises: (1) that attentional selection is in fact enhanced (boosted) following selection of a relevant event (T1) and (2) that attention blocks (bounces) entry of distracting information into working memory. The boosting period is said to take about $150 \mathrm{msec}$ and may recur for additional selection periods by later occurring targets (T2, T3) if they all fall within a previous boosting period. On the other hand, if a distractor is selected during the boosting period, the blocking response typically associated with distractor stimuli is itself amplified, leading to a period of time where most information is now severely suppressed (i.e., the blink itself). In other words, visual input is blocked when it is irrelevant or distracting. When the visual input is really distracting (because it has been accidentally boosted), it produces an ever greater degree of suppression. This newer model of the AB makes no appeal to processing bottlenecks and posits that, although target processing may require limited capacity attentional resources, this constraint is independent of the $\mathrm{AB}$ effect (Olivers, 2007; Olivers, van der Stigchel, \& Hulleman, 2007). Wyble and colleagues have also recently proposed similar boost-followed-by-suppression models of the $A B$, with the main difference being that the suppression is triggered by the encoding into working memory of recently selected events (also, the boosting mechanism is referred to as the blaster; see Bowman \& Wyble, 2007; Wyble, Bowman, \& Nieuwenstein, 2009). We will return to these theories in the General Discussion section, although it should be noted that, although some recent articles have continued to argue for resource depletion models, there seems to be a consensus arising that the filtering models may better capture all aspects of the $\mathrm{AB}$, such as lag-1 sparing and the absence of the $\mathrm{AB}$ when multiple targets are presented sequentially in the RSVP sequence.

In addition to the AB, Ariga and Yokosawa (2008) recently reported another attentional deficit in the processing of rapid events. These authors were investigating the existence of any attentional limitations for the detection of a single target in an RSVP stream when the target appeared early on in the stimulus stream. They proposed that participants would suffer from reduced target-detection accuracy early on in an RSVP task because attentional preparation is required for setting up the visual system to detect brief targets within a rapid sequence of events. Their results indeed showed a cost in the detection of a white letter among blue distractor letters when the target letter appeared early on in an RSVP stream. Performance recovered over a period of about $700-900 \mathrm{msec}$ after the beginning of the RSVP stream and reached ceiling for the remainder of the stream. They named this phenomenon attentional awakening (AA), and they proposed that it resulted from the time taken to orient and configure temporal attention into a state that allows for the identification of rapidly presented visual information (see Figure 1B for the typical time course of this effect). Note that theories regarding the $\mathrm{AB}$ are mute on issues dealing with $\mathrm{T} 1$ processing (such as the AA), because they all focus on accounting for $\mathrm{T} 2$ processing limitations, given that $\mathrm{T} 1$ has 
been successfully processed (e.g., Chun \& Potter, 1995; Jolicœur \& Dell'Acqua, 1998; Shapiro, Raymond, \& Arnell, 1994; R. Ward, Duncan, \& Shapiro, 1996).

However, the literature on foreperiod effects may speak to the phenomenon of the AA. In that literature, it has long been known that the ability to prepare and temporally anticipate a target event can have marked effects on performance (e.g., Niemi \& Näätänen, 1981; Woodrow, 1914). In particular, when there is temporal uncertainty about the target's arrival, performance improves as the foreperiod increases (e.g., Drazin, 1961). The effect typically is explained in terms of temporal contingencies: The chance of the target appearing "now" (given that it has not yet appeared) increases as the time from the start of the trial increases (i.e., as the foreperiod increases), simply because it is known that the target will appear by the end of the trial. More recently, research by Rolke and colleagues has started to look into the mechanisms underlying these preparatory benefits. Directly relevant to the AA, Rolke and Hofmann (2007) linked foreperiod preparation to increased perceptual processing. Rolke (2008) also has shown that foreperiod effects can improve the perception of briefly presented (and masked) letters. And Bausenhart, Rolke, and Ulrich (2008) showed that foreperiod preparation actually improves the temporal resolution of visual perception (in the context of temporal order judgment tasks). Thus, it is possible that underlying the AA may be an ever-increasing temporal resolution of attention as the RSVP unfolds: In other words, it is possible that, as the RSVP progresses, we get better at individuating each letter episode and thus are better able to report them. From this perspective, temporal visual attention might not be entirely unlike temporal auditory attention, which has been proposed to work in brief bursts (or attention pulses) that get locked into rhythmical auditory events (period locking) as well as phase locked to them so as to maximize processing during the onset of these brief events (e.g., Barnes \& Jones, 2000; Large \& Jones, 1999). We will return to this possibility in the General Discussion section.

In the context of the AA, we also note that it is unlikely that foreperiod-driven target anticipation underlies the effect, at least in its simplest form. Ariga and Yokosawa (2008) tested for the contribution of positional foreperiod effects in their Experiments 3 and 4: Prior to the RSVP of letters containing the target, the authors added an RSVP of characters flashing at the same rate and at the same location as the subsequent RSVP containing the target. Despite the presence of this warming signal, an AA was still observed, even though the leading RSVP streams were $600 \mathrm{msec}$ long and the target was now appearing at temporal positions within the overall RSVP stream that should not have produced an AA. Ariga and Yokosawa also tested whether the AA was due to a temporal foreperiod effect by varying the time between fixation and the onset of the RSVP stream within a block of trials. Their results showed an increase of the AA as the foreperiod between fixation and RSVP increased from 0 to 300 to $600 \mathrm{msec}$, the exact opposite pattern of results predicted by temporal foreperiod effects. Even given this finding, it is not entirely clear that the AA does not possess some underlying com- monalities with foreperiod effects. For instance, it is possible that temporal tuning starts only when, or perhaps is contingent upon, the visual system detecting task-relevant stimuli. In other terms, the foreperiod effect on temporal attention might be contingent on task settings, as are other attentional effects (e.g., Folk, Remington, \& Johnston, 1992). If so, the tuning of temporal selection might not start until task-relevant stimuli begin to be perceived. Ariga and Yokosawa's data are consistent with this possibility, because the magnitude of the AA was reduced when the leading RSVP characters were made more similar to letters, which were the task-relevant stimuli (comparing their Experiments 3 and 4). We will come back to this point in the present article.

With all this in mind, it becomes apparent that there is an intriguing similarity between the $\mathrm{AA}$ and the $\mathrm{AB}$, in that both phenomena highlight limitations in our ability to accurately perceive briefly presented targets within a stream of distractors. One goal of this study was to investigate the relationship between these two attentional limitations.

The difficulty in identifying T1 early in RSVP streams (the $\mathrm{AA}$ ) also raises an interesting question regarding its relationship to the $\mathrm{AB}$ and more specifically to the magnitude of the AB. Research has shown mixed results regarding the relation between the difficulty in identifying $\mathrm{T} 1$ (which we call T1 difficulty) and the magnitude of the AB. For instance, studies manipulating the difficulty of the perceptual judgment involved in identifying T1 (achieved through manipulations of backward masking or targetdistractor similarity) have shown no direct relationship between T1 difficulty and the AB (e.g., McLaughlin, Shore, \& Klein, 2001; Shapiro et al., 1994; R. Ward, Duncan, $\&$ Shapiro, 1997). Such results seem to argue against the bottleneck theories of the $\mathrm{AB}$, because longer processing time for $\mathrm{T} 1$ ought to result in longer waiting periods before $\mathrm{T} 2$ can be centrally processed, thereby increasing the likelihood that, and the extent to which, the $\mathrm{T} 2$ representation will be masked. However, a second group of articles have shown an $\mathrm{AB}$ of increased magnitude with increased $\mathrm{T} 1$ difficulty (e.g., Dell'Acqua \& Jolicœur, 2000; Dell'Acqua, Turatto, \& Jolicœur, 2001; Jolicœur, 1998, 1999a, 1999b, 1999c; Jolicœur \& Dell'Acqua, 1998). These latter results appear to be more in line with the traditional bottleneck accounts of the AB. Interestingly, these results have been obtained in the context of increased attentional demands on the T1 task, rather than increased perceptual difficulty of T1 identification. For instance, participants may be asked to report two separate (and separable) attributes of T1 (such as identity and size; see Dell'Acqua \& Jolicœur, 2000; Dell'Acqua et al., 2001; Jolicœur, 1998, 1999a, $1999 b, 1999 c)$ or to report three letters (instead of the usual one) as the T1 task (Jolicœur \& Dell'Acqua, 1998). We discuss the implications of these findings further in the General Discussion, but here it suffices to state that the relationship between $\mathrm{T} 1$ difficulty and the $\mathrm{AB}$ is a vigorously investigated topic and that the qualitative type of manipulation of $\mathrm{T} 1$ difficulty appears to produce different patterns of results: When the perceptual difficulty of $\mathrm{T} 1$ is manipulated, $\mathrm{T} 1$ difficulty does not affect the size of 
the $A B$, whereas attentional manipulations of the $T 1$ task difficulty do seem to modulate the magnitude of the $\mathrm{AB}$ (but see Visser, 2007, for an alternative account of these disparate findings). In light of these results, a second goal of the present study was to investigate whether T1 difficulty, as indexed by the AA effect, would modulate the magnitude of the $\mathrm{AB}$, with the goal of better understanding the mechanisms behind both effects.

To preview, our results showed a relative dissociation of the $\mathrm{AA}$ and $\mathrm{AB}$ phenomena, insofar as factors affecting the magnitude of the AA failed to affect the magnitude of the $A B$, and vice versa. These results suggest that different attentional limitations underlie these two effects. Briefly, we argue that the AA is a stimulus-driven contingent tuning of temporal attention that is dependent on the anticipated length of the RSVP stream: As more and more relevant stimuli are perceived, temporal attention becomes more and more tuned to independently perceiving each instance of these stimuli, up to the point where temporal attention is optimally tuned to the RSVP events. Furthermore, our results show that the speed at which temporal attention is tuned to the stream depends on the anticipated length of the RSVP: In experiments in which participants anticipated longer RSVP streams, larger AA effects were observed than in those in which participants anticipated shorter streams. In that sense, failures to correctly identify $\mathrm{T} 1$ at early stream positions (i.e., the AA) ought to be linked to noisier T1 representations (i.e., the AA is a perceptual effect). In contrast, in the AB, failures to correctly identify $\mathrm{T} 2$, given that $\mathrm{T} 1$ has been processed, ought to be linked to failures to select $\mathrm{T} 2$ from the stream (i.e., the $\mathrm{AB}$ is a filtering effect). These issues will be treated with more detail in the General Discussion.

\section{GENERAL METHOD}

Participants in all experiments performed a one- or two-target letter identification RSVP task, similar to the one-target paradigm of Ariga and Yokosawa (2008). Participants were asked to detect and identify white target letters interspersed among blue distractor letters presented at fixation.

\section{Apparatus and Stimuli}

All experiments were run on 3.4-GHz Pentium IV-based PCs, and stimuli were presented on 17-in. CRT monitors at a resolution of $1,280 \times 1,024$ pixels. Both the blue letters (RGB: 0, 0, 255) and white letters (RGB: 255, 255, 255) were displayed on a gray background (RGB: 128, 128, 128). Each letter subtended approximately $0.6^{\circ} \times 0.6^{\circ}$. A black (RGB: 0, 0, 0) fixation cross briefly appeared at the start of each trial in the center of the screen. Participants sat an average of $57 \mathrm{~cm}$ from the screen.

\section{Procedure}

In all experiments, each trial began with the presentation of a black fixation cross for $494 \mathrm{msec}$, followed by a 329-msec blank interval. Then, a stream of letters was presented rapidly at fixation. All distractor letters were blue, and the targets were white letters presented in the stream. Participants were asked to identify the white targets by pressing the appropriate keys on the keyboard (in any order) after the stream had ended. Each letter was presented on-screen for $24 \mathrm{msec}$, followed by a $71-\mathrm{msec}$ blank interstimulus interval. The letters included every letter of the alphabet except for I, O, Q, and Z and were all uppercase. Although sometimes the same letter could appear twice in a given RSVP stream, it never did so in consecutive posi- tions. In all experiments, participants ran in two conditions: a onetarget identification task and a two-target identification task. The order of these conditions was counterbalanced across participants. In the two-target conditions, the second target letter was presented either two letters after T1 (lag 2) or eight letters after T1 (lag 8), and both conditions were equally likely. The position of the first target in the stream varied for each experiment but was never the first nor the last item in the stream, which meant that $\mathrm{T} 1$ was always forward and backward masked to the same degree at all positions in the RSVP stream. The stream terminated with a presentation of a final distractor letter after the presentation of the final target letter. The next trial began automatically $823 \mathrm{msec}$ after the participant had finished responding. Each condition consisted of three blocks of trials, with a total of six blocks in the experiment. Each condition began with 12 practice trials. Participants were instructed to respond as accurately as possible. All responses were recorded. A $p$ value of .05 was used for all statistical analyses.

\section{EXPERIMENT 1}

In Experiment 1, participants performed both a onetarget and a two-target letter identification task in an RSVP stream. The goal was to replicate the AA effect in both the one- and two-target conditions and to investigate whether the dip in early T1 identification performance would affect accuracy in $\mathrm{T} 2$ identification.

\section{Method}

In Experiment 1, T1 occurred randomly in Positions 2-19 of the RSVP stream. Participants ran in three consecutive blocks of 96 experimental trials (288 total) in each condition (one- vs. two-target conditions) for a total of six blocks (576 trials). The 16 participants received course credit in an undergraduate introduction to psychology course in exchange for their participation in Experiment 1.

\section{Results}

For all analyses, the 18 possible $\mathrm{T} 1$ positions in the RSVP stream were collapsed into six position groups (T1 group), with 3 positions per group, in keeping with the methods of Ariga and Yokosawa (2008). Analysis of the individual position effects did not differ from any of the group position effects. In the two-target condition, T2 was presented either at lag $2(190 \mathrm{msec}$ after T1) or at lag 8 (760 msec after T1), and the magnitude of the AB was measured as the difference in accuracy between these two conditions (lag 8 accuracy minus lag 2 accuracy, given correct T1 identification). As was expected, collapsed across target position in the RSVP stream, performance was significantly worse at lag $2(38.7 \%$ correct $)$ than at lag $8(86.9 \%$ correct $)[t(15)=-9.90, p<.001]$, an $\mathrm{AB}$ magnitude of $48.2 \%$. The magnitude of the $\mathrm{AB}$ was unaffected by $\mathrm{T} 1$ position in the stream, as indexed by $\mathrm{T} 1$ group $[F(5,75)=1.63, p=.16]$; see Figure 2 . The largest visible difference in $\mathrm{AB}$ magnitude occurred between T1 Group 1 and T1 Group 2, yet this difference did not reach significance $[t(15)=1.86, p>.05]$. This pattern of results was identical when $\mathrm{T} 2$ accuracy (rather than $\mathrm{AB}$ magnitude) was used as the dependent measure: no effect of T1 position on T2 accuracy $[F(5,75)=1.18, p>$ $.05]$. In Experiments 2-4, we used $\mathrm{AB}$ magnitude as the dependent measure: All patterns of results were identical regardless of whether $\mathrm{T} 2$ performance or $\mathrm{AB}$ magnitude was used as the dependent measure. 

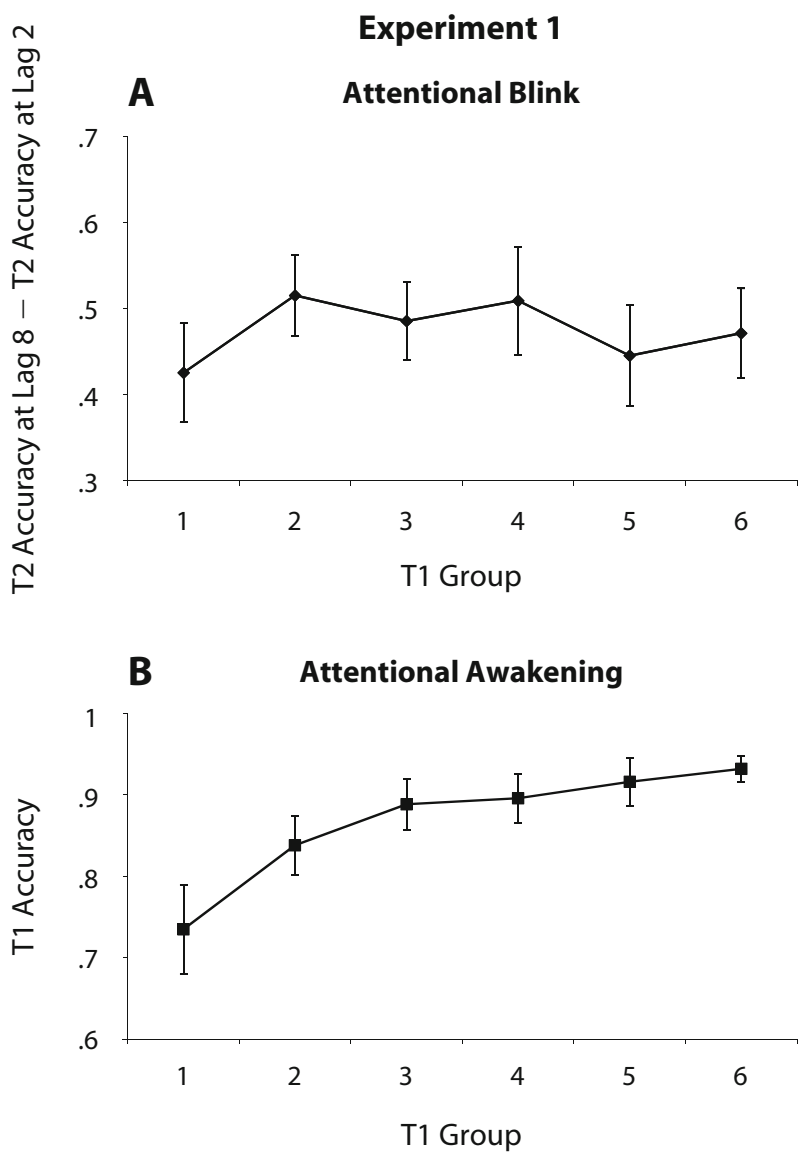

Figure 2. (A) Magnitude of the attentional blink (T2 accuracy at lag $8-\mathrm{T} 2$ accuracy at lag 2 ) in Experiment 1, plotted as a function of its position in the stream (each group represents three sequential target positions (e.g., Group $1=$ Positions 2-4; Group 2 = Positions 5-7). (B) The attentional awakening effect for Experiment 1 in the two-target rapid serial visual presentation condition. Accuracy of T1 identification is plotted as a function of target group.

The AA phenomenon is typically measured by examining differences in $\mathrm{T} 1$ performance observed between target identification accuracy at the beginning of the stream and asymptotic performance toward the end of the stream. In replication of the Ariga and Yokosawa (2008) results, in the single-target condition, performance increased significantly over time from Group 1 to Group 6 [an increase of $12.4 \% ; F(5,75)=19.37, p<.001]$. Planned post hoc comparisons revealed that mean accuracies for Groups 1-3 were significantly lower than those for Groups 4-6 $[t(15)=-5.62, p<.001]$. More specifically, accuracy in Target Group 1 was marginally smaller than that for Group $2[t(15)=-2.04, p=.06]$, which in turn was significantly smaller than accuracy for Group $3[t(15)=$ $-2.62, p<.02]$. Accuracy became asymptotic at $93.3 \%$ correct in Group 6. Further, and more crucial to our study, T1 detection was also affected by target group in the two-target condition, with performance for Group 1 suffering an even larger impairment than in the singletarget condition $[19.7 \% ; F(5,75)=16.001, p<.001]$; see Figure 2. As in the single-target condition, mean accu- racy for Groups 1-3 was significantly lower than that for Groups 4-6 $[t(15)=-4.37, p<.002]$. The effects of the single-target condition were replicated, in that accuracy in Group 1 was worse than accuracy in Group $2[t(15)=$ $-3.94, p<.002]$, which itself was significantly less accurate than Group $3[t(15)=-2.73, p<.02]$. Accuracy became asymptotic at a threshold similar to that in the single-target condition at 93.2\% correct in Group 6 .

Finally, we also tested for processing trade-offs at the early positions. The goal was to test whether incorrect $\mathrm{T} 1$ trials allowed for better T2 identification. In this analysis, we compared T2 performance at lag 2 when T1 was correctly identified with trials in which the $\mathrm{T} 1$ report was incorrect. Only 12 participants had sufficient T1 errors to conduct this analysis (average $N$ per participant was 6.75 for incorrect trials and 16.0 for correct trials). There was no evidence of processing trade-off: Mean T2 accuracy on $\mathrm{T} 1$ correct trials was $33.5 \%$, as compared with $33.9 \%$ on $\mathrm{T} 1$ incorrect trials $[t(11)<1]$.

\section{Discussion}

In Experiment 1, we were able to replicate both the $A B$ and the AA effects and to measure both effects within the same trials. Surprisingly, in the two-target identification task, even though we observed a 20\% drop in T1 accuracy when T1 appeared early in the RSVP stream, this substantial increase in $\mathrm{T} 1$ processing difficulty had no significant effect on the magnitude of the $\mathrm{AB}$. These initial results imply that the processing impairments indexed by the AA and the $\mathrm{AB}$ effects may arise from different loci.

It should be noted that our study differs from several studies that examined the effects of increased T1 difficulty on the $A B$, because we did not use direct perceptual manipulations to induce a drop in T1-related performance, nor did we explicitly manipulate the attentional demands of the $\mathrm{T} 1$ task. We simply used the variability of performance generated by the AA to assess $\mathrm{T} 1$ processing effects on T2. We could argue that, in our study, there was very little difficulty in the perceptual processing of $\mathrm{T} 1$, which was evidenced by asymptotic levels of performance that surpassed 90\% accuracy in Groups 4-6. However, the decrease in T1 performance may have been due to a lowering in the perceptual quality of the T1 signal: If the AA reflects too broad a temporal selection window, the signal representing T1 may in fact be noisier early on in the stream when distractors may be selected alongside the early target. As the temporal tuning of attention becomes narrower, the signal quality of T1 might improve at later positions in the stream because temporal attention may be able to select a single letter at a time from the stream. In that sense then, the AA arises as a perceptual effect, reflecting a poorer representation of T1 at early stream positions. If so, the lack of a significant effect of T1 position on the magnitude of the $\mathrm{AB}$ suggests that different attentional limitations may be responsible for the compromised performance in reporting $\mathrm{T} 1$ and $\mathrm{T} 2$ in these two effects: Whereas the AA may represent a kind of attentional start-up cost involved in tuning the system to better perceive rapid events (i.e., engaging in rapid perception), the $\mathrm{AB}$ may be determined by the presence of perceptual 
filters aimed at all-together blocking distractors that immediately follow the target event.

Experiments 2 and 3 were aimed at further testing the hypothesis of separate attentional loci for these two effects by independently manipulating factors that affect the magnitude of AA (Experiment 2) and the magnitude of the AB (Experiment 3). Finally, Experiment 4 was designed to test the contribution of foreperiod preparation effects on the AA.

\section{EXPERIMENT 2}

In Experiment 2, we manipulated the magnitude of the AA effect. We hypothesized that decreasing the length of the RSVP stream should lead to a smaller AA, because attentional engagement on the streams would be shorter and participants would have a relatively easier time engaging with and preparing for these shorter RSVP streams, as compared with the long RSVP streams used in Experiment 1. Crucially, we predicted that reducing the length of the RSVP should have little to no effect on the magnitude of the $A B$, which should depend only on the factors affecting the T1-induced attentional boosting and subsequent T2 blocking. Additionally, it should be noted that, as in Experiment 1, the actual length of the RSVP on any given trial was always dictated by T1 position and T2 lag (T1 position + T2 lag +1 ), because the RSVP always terminated on the distractor following T2 (as in Experiment 1). In other words, in Experiment 2, we manipulated the range of possible lengths of the RSVP stream, rendering them substantially smaller than the range used in Experiment 1. Finally, it should be noted that Experiment 2 also served as a replication of the null effect of $\mathrm{T} 1$ position on $\mathrm{AB}$ magnitude observed in Experiment 1. If we could replicate this null effect, we would have more support to argue for independent mechanisms underlying the $\mathrm{AA}$ and the $\mathrm{AB}$.

\section{Method}

In Experiment 2, the length of the RSVP stream was shortened, with T1 appearing randomly in Positions 2-13 (the longest trials here were $570 \mathrm{msec}$ shorter than the longest trials in Experiment 1). Participants ran in three sequential blocks of 64 trials (192 total) in the single-target condition and three blocks of 128 trials (384 total) in the two-target condition (a total of 576 trials). As in Experiment 1,16 participants received course credit for their participation in Experiment 2.

\section{Results}

For all analyses, and as in Experiment 1, the 12 possible T1 positions were collapsed into groups of three locationsresulting in four position groups. Analysis of the individual position effects did not differ from the grouped position results. In the single-target condition, as in Experiment 1, target identification accuracy increased with increasing group position $[10.3 \% ; F(3,45)=18.88, p<.001]$. More importantly, in the two-target condition, accuracy in detecting $\mathrm{T} 1$ also increased significantly with increasing group position $[11.2 \% ; F(3,45)=15.51, p<.001]$, but the overall magnitude of the increase (between highest and lowest accuracy for each participant) was less than in Experiment $1[t(30)=2.30, p<.03$; see Figure 3], indicat- ing that decreasing the length of the RSVP stream led to a reduction in the magnitude of the AA effect. Importantly, as in Experiment 1, the magnitude of the $\mathrm{AB}$ effect was not modulated by $\mathrm{T} 1$ position in the RSVP stream $[F(3,45)=$ $1.27, p=.296]$; see Figure 3. Finally, comparing Experiments 1 and 2, there was also no effect of stream length on the magnitude of the $\mathrm{AB}[F(1,150)=1.98, p=.16]$.

\section{Discussion}

In Experiment 2, we observed that shorter RSVP streams produced smaller magnitude AA effects. Yet once again, we failed to observe any evidence of a significant relationship between the accuracy of $\mathrm{T} 1$ report and the magnitude of the $\mathrm{AB}$ at different $\mathrm{T} 1$ positions in the RSVP stream. In addition, stream length did not appear to have any effect on the size of the $\mathrm{AB}$, since the magnitude of the $\mathrm{AB}$ did not differ between Experiments 1 and 2.

In sum, we found evidence that the range of RSVP lengths affects the AA and not the AB: Within the context of shorter RSVP streams, participants find it easier to identify early $\mathrm{T} 1$ targets, than in the context of longer RSVP streams (Experiment 1); yet this factor failed to
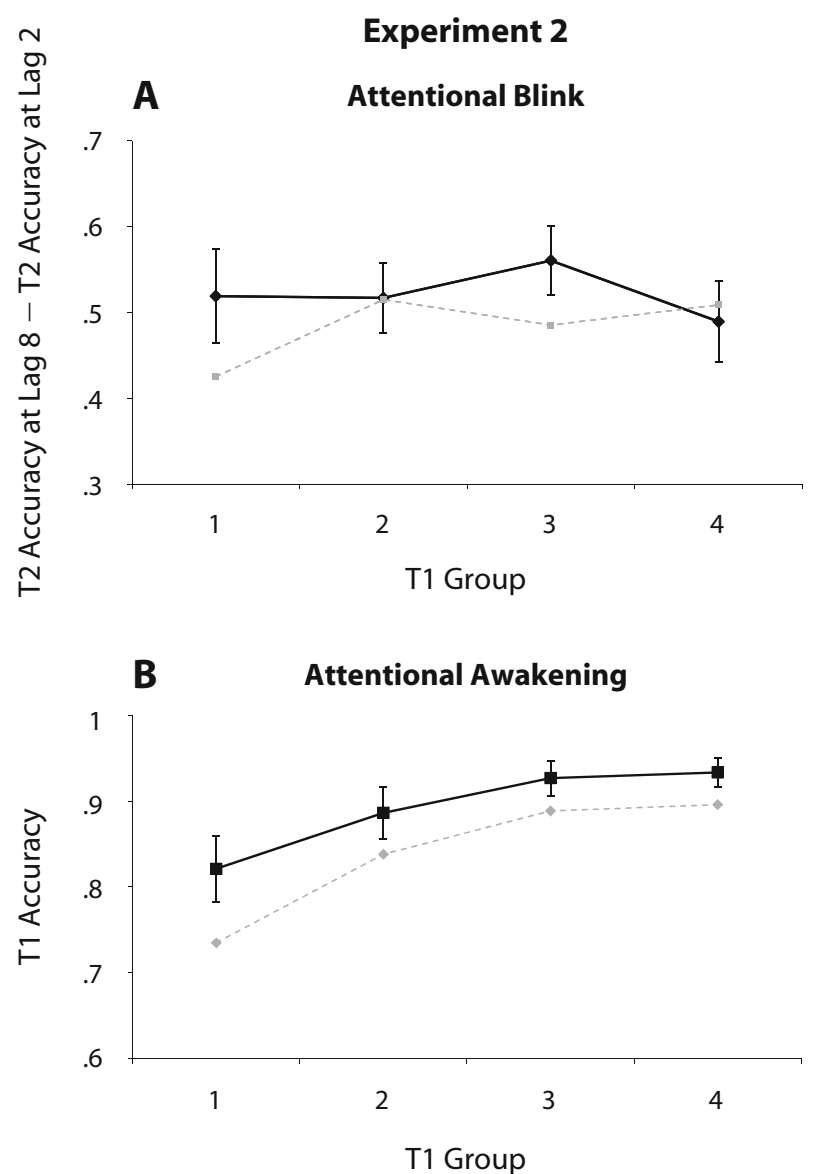

Figure 3. (A) The attentional blink plotted as a function of target group for Experiment 2. (B) The attentional awakening effect for Experiment 2. In both panels, the dotted line shows the results from Experiment 1 for comparison. The maximum length of the target stream was shorter than that in Experiment 1. 
modulate the magnitude of the $\mathrm{AB}$. The position of $\mathrm{T} 1$ in the stream also failed to affect the AB. These results once again suggest that the attentional limitations at play in these two effects are distinct. To test this hypothesis further, in Experiment 3 we investigated whether factors modulating the magnitude of the $\mathrm{AB}$ also produced significant modulations of the AA.

\section{EXPERIMENT 3}

In Experiment 2, the size of the AA effect decreased without a corresponding effect on the size of the AB. In Experiment 3, we attempted to modulate the magnitude of the $\mathrm{AB}$ by decreasing the number of possible positions of T1 within the RSVP stream, while keeping the range of RSVP stream lengths similar to that in Experiment 1. The rationale for this manipulation arose from a basic finding in task-switching studies: that increasing preparation time before a task switch event decreases the magnitude of the task-switching cost (e.g., Altmann, 2004, 2007; Logan, 2003; Monsell, 2003). We figured that perhaps a similar anticipatory effect might help reduce the magnitude of the $\mathrm{AB}$. That is, if participants are provided with expectations about when to expect the T1-T2 target duo, perhaps the blocking of distractors might not be as strong as it would be otherwise, and T2 might be more easily selected from the stream. For this to work, participants would need to have some time to produce and test their temporal expectations. Thus, we reasoned that perhaps reducing the overall number of T1 positions in the stream would help participants estimate whether $\mathrm{T} 1$ has appeared and when to expect it next. From a different perspective, this manipulation might be viewed as inducing a strategic switch in the participants: If they believe that both targets are to appear soon, they might switch from an early one-at-a-time processing strategy to a whole-report strategy, in the hopes of better capturing the few letters left in the stream, which include T2 (see Nieuwenstein \& Potter, 2006, for a demonstration of reduced $\mathrm{AB}$ when the whole-report strategy is used).

In contrast to the expected reduction in $\mathrm{AB}$ magnitude, we predicted that reducing the number of $\mathrm{T} 1$ positions in the stream should have little to no influence on the AA magnitude: If the AA truly reflects a type of attentional setup time during which temporal attention is oriented to the stream and tuned to process rapid visual events for a prolonged period, then the number of $\mathrm{T} 1$ positions should not affect the AA magnitude as long as the overall duration of the streams is preserved.

\section{Method}

In Experiment 3, T1 appeared randomly at Positions 2, 4, 7, 10, 13 , and 16 of the RSVP stream. Participants ran in three sequential blocks of 64 trials (192 total) in the single-target condition and three blocks of 128 trials (384 total) in the two-target condition ( 576 total experimental trials). The 16 participants received course credit for their participation in Experiment 3.

\section{Results and Discussion}

As is evident in Figure 4, reducing the number of possible $\mathrm{T} 1$ positions in the stream did produce a noticeable improvement on the $\mathrm{AB}$ : The magnitude of the $\mathrm{AB}$ decreased with increasing $\mathrm{T} 1$ position $[F(5,75)=2.39, p<$ $.05]$. This negative linear trend (slope of AB magnitude across increasing $\mathrm{T} 1$ position) was significantly different from zero $[t(15)=-1.99, p<.04]$. The size of the AA in the two-target condition (Figure 4) was again significant $(18.2 \%)[F(5,75)=23.36, p<.001]$ and did not differ significantly from the size of the AA effect observed in Experiment $1[t(30)=0.46, p=.651]$, which had a comparable range of RSVP lengths. A comparison between Experiments 2 and 3 also revealed that the magnitude of the AA was smaller in Experiment 2 than in Experiment 3 $[t(30)=2.26, p<.04]$. This difference replicates the difference between Experiments 1 and 2 and further confirms the notion that the range of RSVP stream lengths
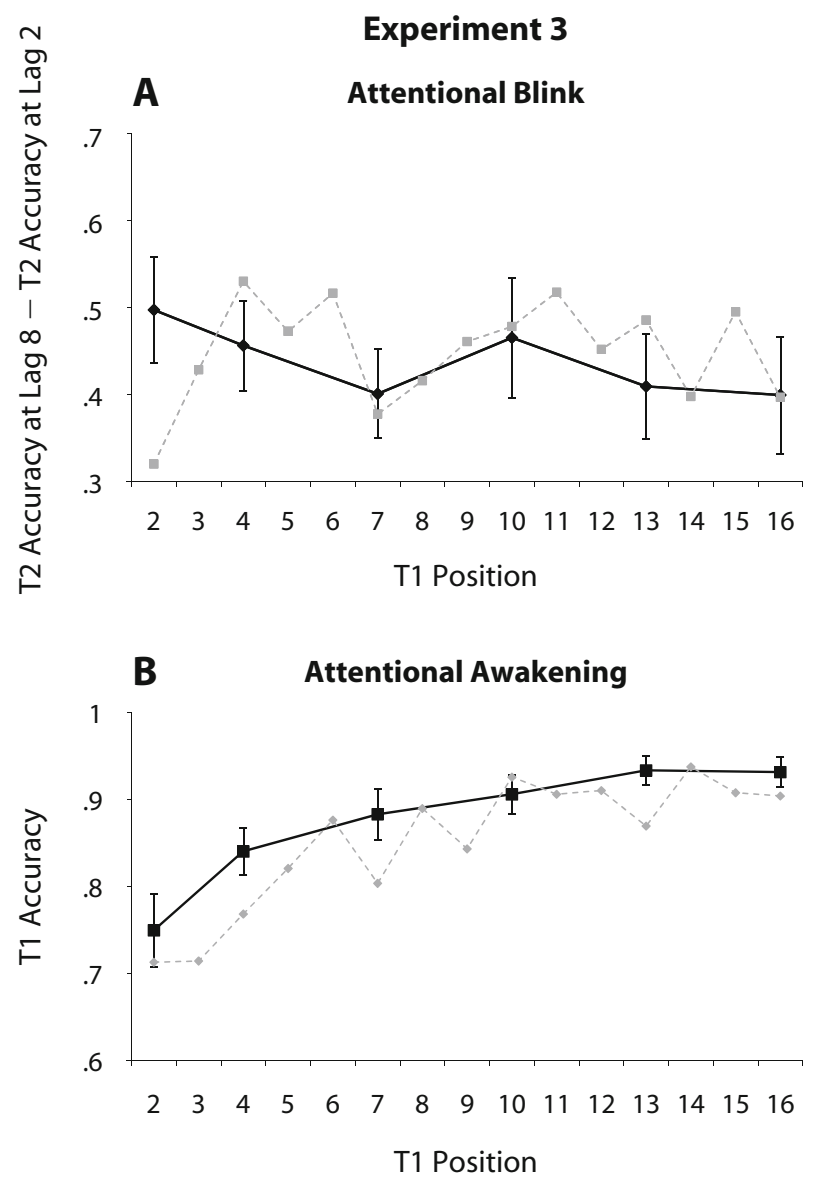

Figure 4. (A) The attentional blink effect for Experiment 3. The position of $\mathrm{T} 1$ in the target stream is constrained to only six possible positions in the entire stream. (B) The attentional awakening effect for Experiment 3. In both panels, the dotted line shows the results from Experiment 1 for comparison. The grouped data from Experiment 1 are broken down into individual position results to match the ungrouped position data from Experiment 3. Error bars for the data from Experiment 1 are not shown, because the lower number of observations per target position yielded large variability at each mean. The average standard error was $6.6 \%$ for the attentional blink data in panel $A$ and $2.2 \%$ for the attentional awakening data in panel $B$. 
influences the magnitude of the AA. It also suggests that attending to RSVP streams that are consistently long requires increased attentional setup costs.

In sum, manipulating the number of possible $\mathrm{T} 1$ positions in an RSVP stream (without altering the length of the stream) had a direct effect on the magnitude of the AB. However, this manipulation failed to modulate the magnitude of the AA, once again suggesting that the AA and the $\mathrm{AB}$ arise from different (temporal) limitations of the attentional system.

\section{EXPERIMENT 4}

Experiment 4 was designed as an investigation into the underlying mechanism of the AA, of which we still know relatively little. As was mentioned in the introduction, Ariga and Yokosawa (2008) tested for the possible contribution of foreperiod effects in the AA, either by adding leading distractors to the RSVP stream (Experiments 3 and 4) or by manipulating the temporal foreperiod between fixation and the onset of the RSVP stream (Experiment 5). However, one could argue that, if an anticipatory effect underlies the AA, this effect may be contingent on the stimuli: When leading distractors consist of stimuli unrelated to the target set (letters), participants may not place any expectations on this section of the stream. That was the case in Ariga and Yokosawa, since in both Experiments 3 and 4 , the leading distractors were not letters (which were used in the RSVP containing the target: a uniquely colored letter). As a result, foreperiod effects, if present, may in fact be contingent on the type of stimuli being viewed.

Here, we tested for foreperiod (or warning) effects on the AA by manipulating the probability that the target would appear at any given position group in the stream. In our previous experiments, as the RSVP progressed, the likelihood of the T1 target's appearing on the next instance of the stream (given that it had not appeared yet) continually increased because the a priori probability of the target's appearing at any position in the stream had been manipulated in pseudorandom fashion (all positions were set to be equally likely). To remedy this, we approximated an exponential probability function to the different target positions, such that now, most T1 targets disproportionately would appear in the first target group position, followed by an exponentially reduced likelihood of its appearing in the second target group position (17\%) or in any subsequent target group position (8\%). An additional advantage of this method is that it increases the number of observations in the early RSVP positions, so that individual position analysis can be performed with more stable estimates of performance.

\section{Method}

In Experiment 4, T1 could appear in Positions 2-19 of the RSVP stream (as was the case in Experiment 1). Participants ran in four sequential blocks of 144 two-target trials (576 total); there was no single-target condition in Experiment 4. In each block, T1 appeared $50 \%$ of the time in the first target group (first three positions), approximately $16.7 \%$ of the time in the second target group, and ap- proximately $8.3 \%$ of the time in each of the third, fourth, fifth, and sixth target groups. In each block, this distribution led to 72 trials in Group 1 (24 counts at each target position), 24 trials in Group 2 (8 counts at each target position), and 12 trials for Groups 3-6 (4 counts at each target position). The 15 participants received course credit for their participation in Experiment 3.

\section{Results}

As in all previous experiments, there was a significant increase $(8.6 \%)$ in $\mathrm{T} 1$ accuracy with increasing group position $[F(5,70)=18.28, p<.001$; see Figure 5], indicating that the AA effect is not entirely dependent on foreperiod effects. However, this magnitude increase was significantly less than that observed in Experiment 1 $[t(29)=2.51, p<.009]$ but not significantly different from that observed in Experiment $2[t(29)=0.857, p=$ $.398]$. T1 accuracy at the beginning of the RSVP stream (Position 2) was also significantly less (14.5\%) than T1 accuracy for Group $4[t(28)=5.01, p<.001]$. In addition, there was a significant downward trend $(14.2 \%)$ in $\mathrm{AB}$ magnitude across group position $[F(5,70)=9.78$, $p<.001]$, which stemmed mainly from a significant decrease in magnitude at the later (and less frequent) T1 groups.
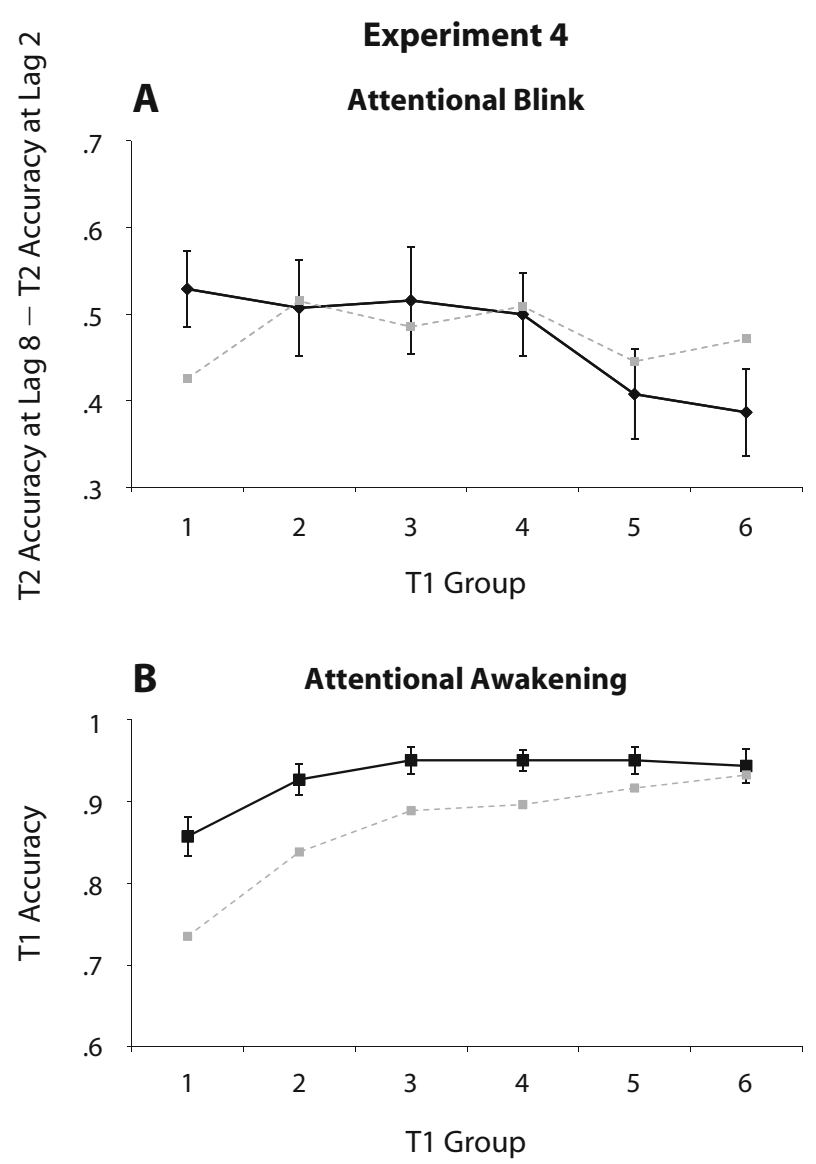

Figure 5. (A) The attentional blink for Experiment 4. (B) The attentional awakening effect for Experiment 4. In both panels, the dotted line shows the results from Experiment 1, for comparison. 


\section{Discussion}

The results of this experiment show that the magnitude of the AA is modulated by the within-stream probability of T1 position. That said, even when, in the vast majority of trials, T1 appeared at the very earliest positions in the RSVP stream, an AA was still observed. The magnitude of the AA, by group, was smaller than the one observed in Experiment 1 (which had comparable total length RSVP streams), yet it was still substantial, as the position analysis showed. It should also be pointed out that the present results suggest that total RSVP length might not be directly related to the magnitude of the AA (as is suggested by cross-experiment comparisons above), but rather, that the AA might be modulated by the expected average of the upcoming RSVP: When on-average longer RSVP streams are expected, larger AAs are observed, and when on-average shorter RSVP streams are expected, smaller AAs are observed. These two variables were confounded when Experiments 1 and 2 were compared, because total RSVP length and expected average length of the stream both varied in the same direction (both decreased) across experiments. In the present experiment, by making T1 much more likely to appear early in the RSVP (while keeping the maximum range of the RSVP as long as in Experiment 1), we effectively produced trials that were on average shorter sequences than those in Experiment 1: The average expected length of the RSVP stream in Experiment 4 was 13 items, with half of the trials having fewer than 10 items, whereas the average expected length of the RSVP in Experiment 1 was 16.5 items. In fact, the expected average length of the stream was closer to that of Experiment 2 (13.5 items). Correspondingly, the magnitude of the AA was also closer to that of Experiment 2 than to that of Experiment 1: In fact, the magnitude of the AA was not significantly different from that in Experiment 2.

In sum, manipulating positional expectancy within the RSVP stream in a pseudoexponential fashion (with very large expectancies early in the stream) did not totally eliminate the AA, contrary to what a simple foreperiod expectancy account would have predicted. That is, even though participants knew to expect T1 within the first 665 msec of the RSVP trial on two thirds of the trials, they still showed a significant start-up cost, finding it difficult to report these early (yet highly likely) targets. In contrast, the magnitude of the AA was comparable to that observed in Experiment 2, strongly suggesting that the actual magnitude of the AA might be determined not only by the temporal position of the target within the stream but also by the anticipated average duration of the RSVP: The more protracted the anticipated average period of sustained temporal attention to the RSVP is, the bigger the magnitude of the AA. ${ }^{1}$

Finally, our manipulation also produced a modulation of the $\mathrm{AB}$ magnitude, with a significant drop in the $\mathrm{AB}$ for the later T1 groups (Groups 5 and 6). Interestingly, this drop appeared in a range of T1 groups in which T1 performance was actually stable (see panel B of Figure 5). In other words, whatever the cause for the reduction in the $\mathrm{AB}$ magnitude was for the later $\mathrm{T} 1$ groups, this cause was likely unrelated to the AA phenomenon itself. It is difficult to say why the AB magnitude dropped for these later groups. We can speculate that perhaps a preparatory effect similar to that observed in Experiment 3 was at play here: For later T1 groups, certainty about the appearance of the T1-T2 duo increases. But why that should be at play here and not in Experiment 1 (which had similar length streams) is hard to say. Perhaps the infrequent nature of these longer sequences allowed participants to better prepare for these late occurring targets, when it became apparent that a longer stream was indeed occurring.

\section{GENERAL DISCUSSION}

The AB corresponds to decrement in performance that arises when two brief targets, appearing close in time, must be both correctly identified. The AA corresponds to decrement in performance that arises when a brief target appearing soon after the beginning of a rapid sequence of events must be correctly identified. Both phenomena are examples of important attentional limitations in vision, insofar as they illustrate impairments in our ability to selectively process a brief event (T2 in the AB, T1 in the AA) that is clearly visible when the attentional constraints are removed (T2 at lag 8 in the AB, T1 at Positions 8 and beyond in the AA). In the present experiments, we sought to determine whether these two attentional effects were related or whether they originated from different attentional limitations. In Experiment 1, both effects were obtained within the same set of trials, and we failed to observe a covariation of $\mathrm{AB}$ magnitude as a function of $\mathrm{T} 1$ difficulty (the AA). This finding suggested that whatever processing limitation was responsible for increasing T1 difficulty at early positions in the RSVP stream was likely to be different from the limitations giving rise to the AB. However, much prior research has shown that increasing T1 difficulty does not necessarily lead to increases in AB magnitude (e.g., McLaughlin et al., 2001; Shapiro et al., 1994; R. Ward et al., 1997), except when the T1 difficulty was increased by increasing attentional demands in the T1 task (e.g., Dell'Acqua \& Jolicœur, 2000; Dell'Acqua et al., 2001; Jolicœur, 1998, 1999a, 1999b, 1999c; Jolicœur \& Dell'Acqua, 1998; but see Visser, 2007, for a different account of why $\mathrm{T} 1$ difficulty may not always relate to $\mathrm{AB}$ magnitude). Therefore, the lack of a T1 difficulty effect on AB magnitude cannot necessarily be taken as evidence that the two phenomena are distinct. Experiments 2 and 3 were aimed at finding manipulations that would selectively modulate one effect (and not the other), in the hopes of building a stronger case that the $\mathrm{AA}$ and the $\mathrm{AB}$ do tell us different things about temporal, selective attention.

Comparing the AA magnitude in Experiment 2 with those in Experiments 1 and 3, we showed that the expected length of the RSVP streams modulates the AA, with longer expected RSVP streams producing larger AAs than shorter expected RSVP streams did. Interestingly, this trend is also consistent with observations from wholereport RSVP tasks, in which participants were asked to report all items in a sequence: In short (up to six-item) sequences, there is no evidence of an AA effect (see Fagot \& Pashler, 1995; Nieuwenstein \& Potter, 2006). In con- 
trast, in Experiment 2, as in Experiment 1, the AB was unaffected by T1 position and by RSVP length. Further, in Experiment 3, we introduced what, to our knowledge, was a novel manipulation of $\mathrm{AB}$ magnitude: We reduced the possible number of T1 locations in the RSVP stream (without reducing the overall length of the streams), predicting that expectations for the target duo T1-T2 would increase as the RSVP stream unfolded. This increased expectation that both $\mathrm{T} 1$ and $\mathrm{T} 2$ would be processed together (rather than one at a time) resulted in an $\mathrm{AB}$ of decreasing magnitude at later $\mathrm{T} 1$ positions. In contrast, reducing the number of possible $\mathrm{T} 1$ positions in the stream did not affect the magnitude of the AA. Finally, we designed Experiment 4 to investigate the extent to which the AA is an aging foreperiod effect by substantially increasing the number of T1s early in the stream. We still found a significant AA (14.5\% when measured at Position 2 in the stream), although T1 performance was quick to become asymptotic (no variations after Group 2). Interestingly, we also found that the AB magnitude was affected at the opposite end of the RSVP stream when T1 performance was stable: AB magnitude decreased in Groups 5 and 6. This result, once again, suggested that different underlying mechanisms must be responsible for the $\mathrm{AA}$ and the $\mathrm{AB}$.

In sum, the present results provide us with clear evidence that the $\mathrm{AA}$ and the $\mathrm{AB}$ represent different limitations of the attentional system. So, what do these results tell us about the underlying mechanisms for these effects?

Let us start with the AB. As was presented in the introduction, there are two classes of theories of the AB. At a first glance, our results seem to be more in line with the filter theories of the $\mathrm{AB}$ than with resource depletion accounts. That follows because, according to resource depletion accounts, the more resources the T1 task takes, the larger the AB should be. Also, if one takes a decrement in $\mathrm{T} 1$ performance as an index of increased T1 processing difficulty, then at the very least, some form of trade-off should be observed between T1 performance and T2 performance. However, in four experiments, we obtained significant modulations of 11 performance (i.e., the AA), yet the AB appeared unaffected by or during those modulations. Thus, one could argue either that the T1 modulation was too weak or that, as on some prior reports, the factors limiting T1 performance in our experiments did not affect the processing bottleneck underlying the AB. With these findings, it is difficult to strongly argue one way or another. In contrast, filter theories of the AB can more easily accommodate this aspect of our results, simply because they are mute with respect to target processing that occurs prior to the moment when the filter blocking T2 is put in place. This follows because, according to these theories, the $\mathrm{AB}$ is a result of blocking of input that is triggered by the appearance of a distractor immediately following $\mathrm{T} 1$ (and not by the processing of T1 itself). From this perspective, it appears clear that T1 processing difficulty ought not to modulate the magnitude of the AB: Whereas T1 might be perceptually processed with varying degrees of success (as we observed during the AA interval), the ability to process T2 at lag 2 is unaffected by the degree of that success, but is affected simply by the fact that $\mathrm{T} 1$ as well as a trailing distractor were detected in the stream. ${ }^{2}$

With respect to our $\mathrm{AB}$ findings, one final issue to discuss is the decrease in $\mathrm{AB}$ magnitude that we observed at later T1 groups in Experiments 3 and 4. This modulation appeared to be independent of the AA, yet it seemed to be telling something important about the nature of the AB. That the strength of the "bouncing" (in Olivers's terms) or suppression of input might be modulated by temporal factors such as the number of target positions and the position of $\mathrm{T} 1$ in the stream is quite interesting. It suggests that either the strength of the bouncing is automatically weakened in these circumstances (when T2 is increasingly likely to appear) or that the participants may be able to soften the strength of the bouncing when there is an increased expectation of the target pair's appearing imminently (or very soon). In any case, our findings suggest that these temporal expectation factors may affect either the strength of the filtering or the speed at which this filtering is put into place. It is impossible to differentiate between these two possibilities at this point, but the finding seems worthy of further investigation.

Regarding the AA, our results showed that the AA performance decrement is unlikely to arise from the same mechanisms leading to the AB deficit. Furthermore, we showed that, when temporal attention is set up to process a stream of rapid events (Ariga \& Yokosawa, 2008), the

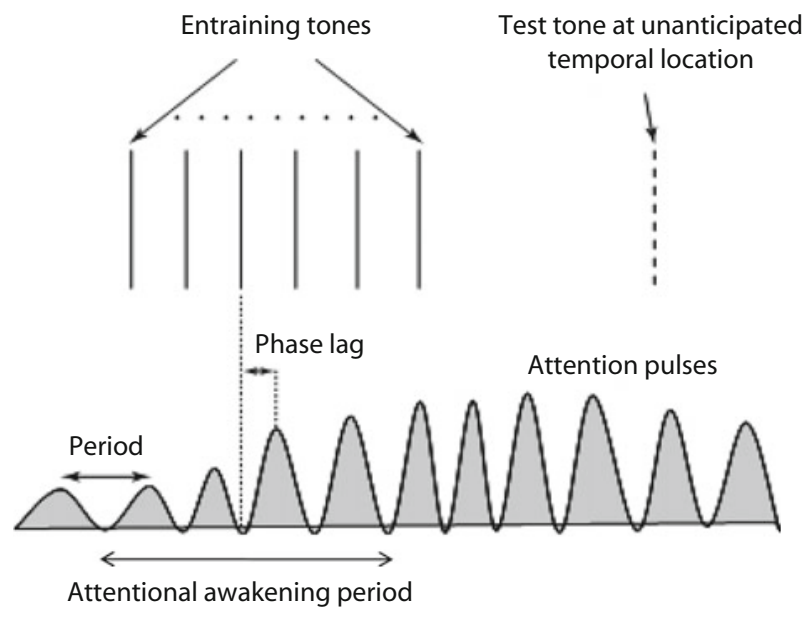

Figure 6. Cartoon of Large and Jones's (1999) theory of oscillatory attention. The oscillations on the bottom represent variations in attentional resources. When in the presence of an entraining stimulus, such as brief, rhythmical tones (represented by slim bars at the top of the figure), the attentional oscillations adopt the same frequency as the entraining stimulus and become phase locked to it. These oscillations become attentional pulses that maximize processing resources at the brief moments when entraining tones are expected. There is also a processing cost that arises when stimulation occurs during an attentional valley. We propose that the AA may reflect the overall lower peaks and longer valleys that are observed before the rapid serial visual presentation stimulation has fully entrained temporal attention (see also Mathewson, Fabiani, Gratton, Beck, \& Lleras, 2009). From "Synchronous Neural Oscillations and Cognitive Processes," by L. M. Ward, 2003, Trends in Cognitive Sciences, 7, p. 557. Copyright 2003 by Elsevier. Adapted with permission. 
expected average length of the stream has an effect on the attentional engagement early on in the stream - such that preparing to view shorter streams appears to be an easier task than preparing for longer streams. This result suggests that the AA is truly a preparatory effect (a setup cost) that arises partly in anticipation of the visual stream, as was initially proposed by Ariga and Yokosawa. That is, the magnitude of the AA was not a factor of the RSVP stream length on a given trial, but of how long it could have been (remember that in our experiments the stream length was dictated by $\mathrm{T} 1$ position in the stream). This finding also provides further evidence that the AA is not due to a type of attentional-switching cost (see Ariga \& Yokosawa, 2008, for converging evidence). Indeed, one could argue that the AA might arise from the switch between attending to fixation (or fairly static events) to attending to rapid events; however, that switch, if present, would be identical for streams of all lengths and would thus predict AA to be unaffected by stream length; yet it is.

So, what might be the cause for this preparatory, startup cost of temporal attention? We believe (in agreement with Ariga \& Yokosawa, 2008) that the AA may reflect the time needed to synchronize attentional pulses to the temporal events in the RSVP stream. As L. M. Ward (2003) argued, one can imagine that, in the absence of any entraining stimulation, attentional resources vary moment to moment. It is speculated that the frequency of these variations (at least in the auditory domain of temporal attention) might be in the order of $0.5-2 \mathrm{~Hz}$. What Large and Jones (1999; see also Barnes \& Jones, 2000) proposed is that, in the presence of entraining stimulation such as a rhythmic beat, attentional resources adopt (if possible) the same periodicity as the entraining stimuli, producing attentional pulses that maximize perceptual processing at very specific moments in time. These attentional pulses also become phase locked to the stimulation, so that most often, the entraining stimuli are encoded at (or near to) the moment when the attentional pulse is at its peak (see Figure 6 for an illustration). Correspondingly, there is a cost to processing events that occur during the dips of these attentional pulses. We believe, then, that the AA may reflect the time it takes for the RSVP stream to drive visual attention into a synchronized (pulsing) state, a state that allows for better individuation and better processing of each letter in the stream (see Mathewson, Fabiani, Gratton, Beck, \& Lleras, 2009, for evidence regarding this last point).

If we take the view that the AA may reflect an attentional synchronization to the stimulus events, then we can nicely summarize our findings and the dissociation we obtained between the $\mathrm{AA}$ and the $\mathrm{AB}$ as follows: The $\mathrm{AA}$ is about the fetching, whereas the $\mathrm{AB}$ is about the blocking, of information. Early on in an RSVP stream, the fetching is subpar: Many letters appear before attention tunes into the rhythm of the visual events - that is, before attention pulses in synchrony with those events. As a result, early targets enjoy comparatively fewer processing resources than later targets do, giving rise to the AA. On the other hand, the $\mathrm{AB}$ occurs because the fetcher of information has fetched a target and a distractor from the stream. To protect $\mathrm{T} 1$ processing and consolidation, a blocking filter is put in place that suppresses processing resources for about $500 \mathrm{msec}$. It is this blocking or suppression that gives rise to the $\mathrm{AB}$.

Lastly, with respect to the AA, our results also showed that the speed of attentional tuning is variable: When participants anticipate long entraining stimuli streams, attentional tuning is slower (and the AA is more protracted) than when participants anticipate relatively shorter entraining stimuli streams. Several testable hypotheses come out of this report: What would happen if the RSVP stream were irregularly timed? What would happen if the distractor following $\mathrm{T} 1$ were presented during an attentional valley? How would this affect the magnitude of the AB? We are currently working on these and related issues in our lab. We hope that, with this report, we have helped to illustrate that the AA is a nontrivial effect, worthy of future investigation.

\section{AUTHOR NOTE}

The authors thank Vince Di Lollo and Mark Nieuwenstein for their constructive feedback on the manuscript. Address correspondence to A. Lleras, Department of Psychology, University of Illinois at UrbanaChampaign, 603 E. Daniel St., Champaign, IL 61820 (e-mail: alejandro .1leras@gmail.com).

\section{REFERENCES}

Altmann, E. M. (2004). Advance preparation in task switching: What work is being done? Psychological Science, 15, 616-622. doi:10.1111/j.0956-7976.2004.00729.x

Altmann, E. M. (2007). Comparing switch costs: Alternating runs and explicit cuing. Journal of Experimental Psychology: Learning, Memory, \& Cognition, 33, 475-483. doi:10.1037/0278-7393.33.3.475

Ariga, A., \& YokosaWA, K. (2008). Attentional awakening: Gradual modulation of temporal attention in rapid serial visual presentation. Psychological Research, 72, 192-202. doi:10.1007/s00426-006 $-0100-4$

Barnes, R., \& Jones, M. R. (2000). Expectancy, attention, and time. Cognitive Psychology, 41, 254-311. doi:10.1006/cogp.2000.0738

Bausenhart, K. M., Rolke, B., \& Ulrich, R. (2008). Temporal preparation improves temporal resolution: Evidence from constant foreperiods. Perception \& Psychophysics, 70, 1504-1514. doi:10.3758/ PP.70.8.1504

Bowman, H., \& Wyble, B. (2007). The simultaneous type, serial token model of temporal attention and working memory. Psychological Review, 114, 38-70. doi:10.1037/0033-295X.114.1.38

Chun, M. M., \& PotTer, M. C. (1995). A two-stage model for multiple target detection in rapid serial visual presentation. Journal of Experimental Psychology: Human Perception \& Performance, 21, 109-127. doi:10.1037/0096-1523.21.1.109

Dell'Acqua, R., \& Joliceur, P. (2000). Visual encoding of patterns is subject to dual-task interference. Memory \& Cognition, 28, 184-191.

Dell'Acqua, R., Turatto, M., \& Joliceeur, P. (2001). Cross-modal attentional deficits in processing tactile stimulation. Perception \& Psychophysics, 63, 777-789.

Di Lollo, V., Kawahara, J.-I., Ghorashi, S. M. S., \& EnNs, J. T. (2005). The attentional blink: Resource limitation or temporary loss of control? Psychological Research, 69, 191-200. doi:10.1007/s00426 $-004-0173-\mathrm{x}$

DrazIN, D. H. (1961). Effects of foreperiod, foreperiod variability, and probability of stimulus occurrence on simple reaction time. Journal of Experimental Psychology, 52, 43-50.

Dux, P. E., Asplund, C. L., \& Marois, R. (2008). An attentional blink for sequentially presented targets: Evidence in favor of resource depletion accounts. Psychonomic Bulletin \& Review, 15, 809-813. 
Dux, P. E., Asplund, C. L., \& Marois, R. (2009). Both exogenous and endogenous target salience manipulations support resource depletion accounts of the attentional blink: A reply to Olivers, Spalek, Kawahara, \& Di Lollo (2009). Psychonomic Bulletin \& Review 16, 219-224.

Dux, P. E., Ivanoff, J. G., Asplund, C. L., \& Marois, R. (2006). Isolation of a central bottleneck of information processing with timeresolved fMRI. Neuron, 52, 1109-1120.

Fagot, C., \& Pashler, H. (1995). Repetition blindness: Perception or memory failure? Journal of Experimental Psychology: Human Perception \& Performance, 21, 275-292. doi:10.1037/0096-1523.21.2.275

Folk, C. L., Remington, R. W., \& Johnston, J. C. (1992). Involuntary covert orienting is contingent on attentional control settings. Journal of Experimental Psychology: Human Perception \& Performance, 18, 1030-1044. doi:10.1037/0096-1523.18.4.1030

Giesbrecht, B. L., \& Di Lollo, V. (1998). Beyond the attentional blink: Visual masking by object substitution. Journal of Experimental Psychology: Human Perception \& Performance, 24, 1454-1466. doi:10.1037/0096-1523.24.5.1454

JOLICEUR, P. (1998). Modulation of the attentional blink by on-line response selection: Evidence from speeded and unspeeded Task $\mathrm{deci}_{1}$ sions. Memory \& Cognition, 26, 1014-1032.

Jolicceur, P. (1999a). Concurrent response-selection demands modulate the attentional blink. Journal of Experimental Psychology: Human Perception \& Performance, 25, 1097-1113. doi:10.1037/0096 $-1523.25 .4 .1097$

JOLICCEUR, P. (1999b). Dual-task interference and visual encoding. Journal of Experimental Psychology: Human Perception \& Performance, 25, 596-616. doi:10.1037/0096-1523.25.4.1097

Jolicceur, P. (1999c). Restricted attentional capacity between sensory modalities. Psychonomic Bulletin \& Review, 6, 87-92.

Jolicceur, P., \& Dell'ACQua, R. (1998). The demonstration of shortterm consolidation. Cognitive Psychology, 36, 138-202.

LARge, E. W., \& Jones, M. R. (1999). The dynamics of attending: How people track time-varying events. Psychological Review, 106, 119159. doi:10.1037/0033-295X.106.1.119

Logan, G. D. (2003). Executive control of thought and action: In search of the wild homunculus. Current Directions in Psychological Science, 12, 45-48. doi:10.1111/1467-8721.01223

Mathewson, K. E., Fabiani, M., Gratton, G., Beck, D. M., \& LleRAS, A. (2009). Making waves in the stream of consciousness: Eliciting predictable oscillations in visual awareness with pre-target entrainment at $12 \mathrm{~Hz}$. Manuscript submitted for publication.

Mclaughlin, E. N., Shore, D. I., \& Klein, R. M. (2001). The attentional blink is immune to masking-induced data limits. Quarterly Journal of Experimental Psychology, 54A, 169-196. doi:10.1080/02724980042000075

Monsell, S. (2003). Task switching. Trends in Cognitive Sciences, 7, 134-140. doi:10.1016/S1364-6613(03)00028-7

Niemi, P., \& NÄÄtänen, R. (1981). Foreperiod and simple reaction time. Psychological Bulletin, 89, 133-162. doi:10.1037/0033 $-2909.89 .1 .133$

Nieuwenstein, M. R., \& Potter, M. C. (2006). Temporal limits of selection and memory encoding: A comparison of whole versus partial report in rapid serial visual presentation. Psychological Science, 17, 471-475. doi:10.1111/j.1467-9280.2006.01730.x

Olivers, C. N. L. (2007). The time course of attention: It is better than we thought. Current Directions in Psychological Science, 16, 11-15. doi:10.1111/j.1467-8721.2007.00466.x

Olivers, C. N. L., \& Meeter, M. (2008). A boost and bounce theory of temporal attention. Psychological Review, 115, 836-863.
Olivers, C. N. L., van der Stigchel, S., \& Hulleman, J. (2007). Spreading the sparing: Against a limited-capacity account of the attentional blink. Psychological Research, 71, 126-139. doi:10.1007/ s00426-005-0029-Z

RaYmond, J. E., Shapiro, K. L., \& Arnell, K. A. (1992). Temporary suppression of visual processing in an RSVP task: An attentional blink? Journal of Experimental Psychology: Human Perception \& Performance, 18, 849-860. doi:10.1037/0096-1523.18.3.849

Rolke, B. (2008). Temporal preparation facilitates perceptual identification of letters. Perception \& Psychophysics, 70, 1305-1313. doi:10.3758/PP.70.7.1305

Rolke, B., \& Hofmann, P. (2007). Temporal uncertainty degrades perceptual processing. Psychonomic Bulletin \& Review, 14, 522-526.

Shapiro, K. L., Raymond, J. E., \& Arnell, K. M. (1994). Attention to visual pattern information produces the attentional blink in rapid serial visual presentation. Journal of Experimental Psychology: Human Perception \& Performance, 20, 357-371. doi:10.1037/0096 $-1523.20 .2 .357$

Visser, T. A. W. (2007). Masking T1 difficulty: Processing time and the attentional blink. Journal of Experimental Psychology: Human Perception \& Performance, 33, 285-297. doi:10.1037/0096-1523.33.2.285

WARD, L. M. (2003). Synchronous neural oscillations and cognitive processes. Trends in Cognitive Sciences, 7, 553-559. doi:10.1016/j tics.2003.10.012

Ward, R., Duncan, J., \& Shapiro, K. L. (1996). The slow time-course of visual attention. Cognitive Psychology, 30, 79-109. doi:10.1006/ cogp.1996.0003

Ward, R., Duncan, J., \& Shapiro, K. L. (1997). Effects of similarity, difficulty, and nontarget presentation on the time course of visual attention. Perception \& Psychophysics, 59, 593-600.

Woodrow, H. (1914). The effect upon reaction time of variation in the preparatory interval. In J. Angell, H. Warren, J. Watson, \& S. Franz (Eds.), The psychological monographs (pp. 16-65). Princeton, NJ: Psychological Review Co.

Wyble, B., Bowman, B., \& Nieuwenstein, M. (2009). The attentional blink provides episodic distinctiveness: Sparing at a cost. Journal of Experimental Psychology: Human Perception \& Performance, 35, 787-807.

\section{NOTES}

1. Although the direction of this effect was evident in both the singleand dual-target conditions, we only had power to test the differences between experiments in the dual-target conditions. Thus, it is possible that this conclusion may apply only to trials in which several items appear after T1 (as is the case in dual-target streams) and may be less of an issue when only one item follows T1 (as is the case in single-target streams). We thank an anonymous reviewer for pointing out this potential confound.

2. This is not to say that bottleneck effects can never be triggered by the $\mathrm{T} 1$ task but merely that the trigger for the $\mathrm{AB}$ is the presence of the bounce filter rather than the processing of T1 itself. In other words, if one designs $\mathrm{T} 1$ tasks that sufficiently tax cognitive and memory processes (as has been done by Dell'Acqua \& Jolicœur, 2000, and Jolicœur, 1998, 1999a, 1999b, 1999c), one can expect even worse performance at the T2 task than what would be observed with $\mathrm{AB}$ blocking alone. But this worsening of performance does not necessarily reflect underlying $\mathrm{AB}$ mechanisms, but overall resource-limited performance.

(Manuscript received March 24, 2008; revision accepted for publication May 2, 2009.) 